Revue d'histoire de l'enfance " irrégulière »

Le Temps de l'histoire

15 | 2013

Enfances déplacées. (II) en temps de guerre

\title{
Négocier l'aide humanitaire : les évacuations d'enfants espagnols vers la France pendant la guerre civile (1936-1939)
}

Negotiating humanitarian aid. The evacuation of Spanish children to France during the civil war (1936-1939)

\section{Célia Keren}

\section{OpenEdition}

Journals

Édition électronique

URL : http://journals.openedition.org/rhei/3515

DOI : 10.4000/rhei.3515

ISSN : $1777-540 \mathrm{X}$

Éditeur

Presses universitaires de Rennes

Édition imprimée

Date de publication : 30 octobre 2013

Pagination : 167-183

ISBN : 978-2-7535-2896-3

ISSN : $1287-2431$

Référence électronique

Célia Keren, « Négocier l'aide humanitaire : les évacuations d'enfants espagnols vers la France pendant la guerre civile (1936-1939) », Revue d'histoire de l'enfance « irrégulière » [En ligne], 15 | 2013, mis en ligne le 30 octobre 2015, consulté le 19 avril 2019. URL : http://journals.openedition.org/ rhei/3515; DOI : 10.4000/rhei.3515

Ce document a été généré automatiquement le 19 avril 2019

(C) PUR 


\title{
Négocier l'aide humanitaire : les évacuations d'enfants espagnols vers la France pendant la guerre civile (1936-1939)
}

\author{
Negotiating humanitarian aid. The evacuation of Spanish children to France \\ during the civil war (1936-1939)
}

Célia Keren

\section{Introduction}

1 Pendant la guerre civile espagnole (juillet 1936-avril 1939), près de trente mille enfants sont évacués de la zone républicaine de l'Espagne vers des pays étrangers. Environ la moitié part vers la France. Pour une période allant de quelques mois à plusieurs années, ils y sont pris en charge, hébergés dans des familles ou des colonies collectives, et scolarisés. Ce déplacement massif et organisé de population enfantine s'ajoute aux flux périodiques de réfugiés fuyant les combats. Il est déclenché par un accord, en novembre 1936, entre le gouvernement républicain, du côté espagnol, et la Confédération générale du travail (CGT), côté français. D'autres acteurs s'agrègent par la suite à ce programme - ou cherchent à le concurrencer en montant des comités rivaux. Cependant, jusqu'à la fin de la guerre civile, la CGT continue, à travers son Comité d'accueil aux enfants d'Espagne (CAEE), à superviser la majorité des dix à quinze mille enfants espagnols évacués à un moment donné vers le territoire français ${ }^{1}$.

Dans le cadre de l'émergence des États-nations européens au XIX siècle, l'enfant est de plus en plus considéré comme l'une des clés de la puissance future, et donc un enjeu politique. Dans la France du tournant du $\mathrm{xx}^{\mathrm{e}}$ siècle, par exemple, tant les politiques sociales que scolaires sont orientées vers l'accroissement et l'amélioration du patrimoine humain de la Nation, dans le cadre de la rivalité avec l'Allemagne. De même, dans la 
Bohême disputée entre nationalistes tchèques et allemands, la protection sociale et l'école sont les moyens privilégiés d'une lutte acharnée pour l'appropriation nationale des enfants ${ }^{2}$. Le meilleur moyen de «faire » des Français, des Allemands, des Tchèques, etc., n'est-il pas de les prendre dès le plus jeune âge ${ }^{3}$ ? Il n'est donc pas étonnant que les guerres du premier $\mathrm{xx}^{\mathrm{e}}$ siècle, qui ont touché les populations civiles avec une ampleur inédite, aient poussé les États à protéger particulièrement les enfants, que ce soit par des politiques de protection sociale qui se poursuivront en temps de paix, ou par des opérations de sauvetage ad hoc. Ainsi, des évacuations enfantines, concernant parfois des dizaines de milliers d'enfants, sont organisées à plusieurs reprises en Europe entre 1914 et 1945 , pour les mettre à l'abri de la faim et des violences guerrières ${ }^{4}$. Pendant la guerre d'Espagne, des enfants de Malaga, Tolède et Madrid sont évacués vers les zones orientales de la péninsule, éloignées des combats. Dans le cadre du mouvement international de solidarité envers la République espagnole, des groupes d'enfants sont également envoyés passer les années de guerre à l'étranger. Mais cet éloignement risque de distendre les liens entre l'État-nation espagnol et « ses » enfants. Il fait aussi surgir le spectre de leur «dénationalisation». Dès lors, la relève de l'État providence espagnol par l'aide humanitaire ne se fait pas à n'importe quelles conditions.

3 Cet article porte sur les modalités négociées (et parfois contestées) entre le gouvernement espagnol et la CGT, de l'évacuation des enfants espagnols vers la France au cours de la guerre civile. Par là, il s'inscrit dans une réflexion plus large sur l'histoire de l'action humanitaire. Celle-ci est encore mal connue, malgré l'importance du phénomène dans nos sociétés contemporaines 5 . Elle a longtemps été victime d'une certaine myopie chronologique, qui la faisait débuter après la seconde guerre mondiale ou, pire, dans les années 1970. Dès lors, les époques précédentes se sont trouvées rejetées dans une "préhistoire ${ }^{6}$ " de l'humanitaire. Mais depuis quelques années, une nouvelle historiographie insiste sur l'importance du xix ${ }^{e}$ siècle et la transition provoquée par le premier conflit mondial vers un « humanitarisme moderne ${ }^{7}$ » qui se consacre en priorité à l'enfance. En effet, jusque-là, l'humanitaire se consacre à la réglementation des violences sur le champ de bataille et aux secours aux soldats blessés et prisonniers de guerre. Il s'institutionnalise dans le Comité international de la Croix-Rouge (CICR) et dans les conventions de Genève et La Haye de 1906-1907. Mais la Grande Guerre et ses lendemains voient l'émergence d'un nouveau type d'humanitaire, qui prend acte des effets de la guerre totale et s'intéresse aux civils - mais pas à n'importe quels civils. Un membre du CICR résume bien cette évolution après une entrevue en 1920 avec Eglantyne Jebb, fondatrice de la première organisation humanitaire d'aide aux enfants, l'Union internationale de secours aux enfants : «Il faut enfin que nous puissions proclamer que la guerre qui s'arrête devant le combattant blessé doit encore bien davantage respecter l'enfant innocent ${ }^{9}$.»

4 Le deuxième apport de cette historiographie récente est d'avoir mis au jour les liens ambigus entretenus, dès ses origines, par ce nouvel humanitarisme avec les derniers feux du colonialisme. Ces liens s'observent à deux niveaux. D'une part se logent, au cœur des premières actions internationales d'aide à l'enfance, des rapports de force géopolitiques inégaux. Keith Watenpaugh montre par exemple combien le sauvetage par la Société des Nations des enfants arméniens déportés et séquestrés pendant le génocide n'a été possible que grâce à une situation de quasi-occupation de l'ancien Empire ottoman. L'humanitarisme, en cela, dépend autant qu'il accentue des relations structurellement asymétriques, concrétisées ici par la perte de souveraineté presque totale du pays 
récipiendaire de l'aide ${ }^{10}$. D'autre part, le contenu même des projets de protection de l'enfance vise souvent à modifier les normes et comportements des populations et familles secourues. Au début des années 1920, certains travailleurs humanitaires américains n'hésitent pas à décrire, par exemple, les Européens de l'Est qu'ils secourent comme « des idiots et des crétins ${ }^{11}$ », et les mœurs des familles françaises de «barbares ${ }^{12}$ ». En ce sens, les politiques humanitaires de l'entre-deux-guerres héritent manifestement d'une partie du langage, des pratiques et des rapports de force internationaux associés à la mission civilisatrice des puissances coloniales - y compris lorsque ces politiques sont destinées, par une étrange ironie du sort, à ces anciennes puissances elles-mêmes.

Or, l'action lancée à partir de novembre 1936 par le Comité d'accueil aux enfants d'Espagne présente l'intérêt, il nous semble, d'éclairer un autre aspect de l'humanitaire de l'entre-deux-guerres et de montrer par là sa complexité. En effet, ici, l'action humanitaire ${ }^{13}$ ne se déploie pas au sein d'une relation asymétrique quasi-coloniale : au contraire, le gouvernement espagnol jaloux de ses prérogatives négocie et, parfois, refuse les évacuations d'enfants lorsqu'elles ne remplissent pas les conditions voulues. En cela, il résiste à tout empiètement sur sa souveraineté, jusqu'à tenter de transformer l'aide humanitaire en prolongement des politiques sociales et éducatives nationales qu'il cherche à développer. Comment se joue, dès lors, le rapport de force entre dispensateur et bénéficiaire de l'aide ? Et que révèle-t-il des multiples virtualités de l'humanitaire de l'entre-deux-guerres?

6 Pour aborder ces questions, nous nous appuyons sur des documents d'archives privées et publiques françaises et espagnoles (déposées à l'Institut d'histoire sociale de la CGT, aux Archives nationales, à la Bibliothèque internationale de documentation contemporaine et à l'Archivo General de la Administración) ainsi que sur la presse militante syndicale française. L'analyse portera d'abord sur les modalités de la négociation et renégociation de l'aide humanitaire entre la CGT et le gouvernement espagnol. Nous décrirons ensuite comment les rapports de force entre acteurs se reflètent dans les choix des lieux, des structures d'accueil et des solutions pédagogiques pour les enfants espagnols accueillis en France entre 1936 et 1939.

\section{La négociation du contrat humanitaire}

\section{L'établissement du contrat, novembre-décembre 1936}

7 En raison de sa situation géographique, la France voit arriver très vite les premiers réfugiés de la guerre d'Espagne. Des initiatives locales d'aide émergent dans leur sillage, surtout en direction des enfants. Entre août et octobre 1936, certaines organisations de secours, pour la plupart liées au conglomérat communiste, élaborent les premiers projets en vue d'organiser une évacuation d'enfants espagnols vers la France - sans succès ${ }^{14}$. Une opération de ce type, structurée et de grande ampleur, ne commence qu'en décembre 1936, après que la CGT a lancé le Comité d'accueil aux enfants d'Espagne (CAEE) ${ }^{15}$.

8 Cet organisme est le fruit de la rencontre ou, plus précisément, d'allers-retours entre sollicitations et propositions émises de part et d'autre des Pyrénées. L'idée en est d'abord proposée par les responsables de la Ligue espagnole des droits de l'homme de passage à Paris afin de plaider la cause de la République. Elle est bien accueillie par la CGT, notamment par les responsables ex-confédérés en contact avec la Ligue française des 
Droits de l'homme. Ce sont ces hommes qui décident de créer, en novembre 1936, le CAEE. Leur projet obtient rapidement l'accord du gouvernement de Léon Blum ${ }^{16}$. Plus importante est cependant la coordination avec les autorités espagnoles républicaines qui, seules, peuvent autoriser et organiser la sortie des enfants vers la France. Une réunion est organisée en décembre à Paris avec Mercedes Maestre, sous-secrétaire à la Santé et l'Assistance sociale du ministère dirigé par Federica Montseny. En résultent neuf « Indications pour la venue en France des enfants d'Espagne ${ }^{17}$ ». Un brouillon manuscrit de ce texte, dans les archives de la CGT, révèle par ses nombreux hispanismes, qu'il a d'abord été rédigé par une main espagnole, peut-être par la sous-secrétaire elle-même, en préparation ou pendant la réunion avec la $\mathrm{CGT}^{18}$.

9 Ces neuf «Indications » font l'objet d'une circulaire confédérale à toutes les fédérations nationales de la CGT et unions départementales des syndicats, chargées de créer des souscomités d'accueil ${ }^{19}$. Elles ont donc valeur règlementaire au sein du mouvement syndical. La CGT accorde une très grande valeur à cet accord. Dans les articles publiés dans son quotidien, Le Peuple, elle rappelle inlassablement l'«accord entre le gouvernement espagnol et le Comité d'accueil aux enfants d'Espagne ${ }^{20}$ », le "plein accord avec le gouvernement républicain de l'Espagne ${ }^{21} »$, le "parfait accord avec la sous-secrétaire d'État à la Santé2 ${ }^{22}$ ». Dans un seul article de juillet 1937, il est fait mention de l'« accord complet", du "commun accord", puis à nouveau de l'«accord complet avec le gouvernement espagnol ${ }^{23}$ ». Un article du mois suivant souligne encore la «liaison avec les représentants du gouvernement espagnol », concluant: " Toutes ces actions, nous le répétons, se sont accomplies en complet accord avec le gouvernement espagnol ${ }^{24}$. » La CGT ne se paye pas de mots. À deux reprises au moins, en décembre 1936 et en janvier 1937, lui sont adressées des demandes individuelles d'accueil d'enfants espagnols en France. Dans les deux cas elle oppose une fin de non-recevoir, dans des termes à peu près identiques :

«Nous ne prenons que les enfants qui nous sont envoyés directement par le Gouvernement de Madrid, ainsi qu'il résulte des conventions que nous avons avec ce dernier »; « Par suite des accords que nous avons passés avec le gouvernement espagnol, notre comité doit assurer seulement l'hébergement des enfants venus d'Espagne et à nous adressés par le gouvernement ${ }^{25} »$.

\section{Premiers amendements au contrat initial}

10 Or, en avril 1937, après seulement quatre mois, la ministre Federica Montseny amende unilatéralement les termes du contrat: "Les accords antérieurs correspondaient à l'époque au point de vue du gouvernement; actuellement il en a changé dans le sens d'accepter uniquement le régime en colonies pour les enfants évacués à l'étranger ${ }^{26}$.» $\mathrm{Ce}$ revirement est loin d'être cosmétique. Comme le fait remarquer le secrétaire général du CAEE, Georges Buisson, la difficulté de trouver des lieux d'hébergement adéquats et les « dépenses considérablement plus élevées » qu'impose le régime collectif, par rapport au placement familial, fragilisent toute l'opération ${ }^{27}$. La ministre espagnole en est bien consciente, et confirme: "En ce moment nous préférons diminuer le nombre d'expéditions que de placer [les enfants] en régime familial ${ }^{28}$ ».

Les archives sont muettes sur la manière dont a été négocié l'accord initial de décembre 1936. Mais les courriers échangés début avril 1937 révèlent deux points importants sur les modalités de l'aide humanitaire apportée par la CGT. Tout d'abord, le gouvernement espagnol n'en est pas dépendant et il n'hésite pas à prendre le risque de la 
voir perdre en efficacité, et même se tarir, si elle ne correspond pas à ses besoins tels que lui seul les définit et redéfinit. Ici, c'est bien le gouvernement espagnol qui établit le cahier des charges humanitaires et qui décide si le CAEE, ou tout autre organisme, y répond. Ainsi, en février 1937, le gouvernement de Valence refuse l'offre d'un comité suisse d'accueillir 500 enfants espagnols, précisément parce qu'il n'est pas satisfait des conditions offertes ${ }^{29}$. En juin de la même année, malgré les besoins d'hébergement considérables créés par la chute du front Nord et le départ de dizaines de milliers de réfugiés, la même proposition est à nouveau rejetée, quoique « le seul point sur lequel [le comité suisse et le gouvernement basque de Bilbao] ne tomb[ent] pas d'accord est l'accompagnement des enfants par 2-3 maîtresses d'école espagnoles ${ }^{30}$ ».

Deuxième point, la CGT accepte le revirement du gouvernement espagnol sans regimber. Lorsqu'elle entend dire que celui-ci «ne se montrerait plus favorable au placement dans les familles effectué dans les conditions [...] envisagées en commun », elle demande des "précisions » avec une extrême déférence :

« nous nous excusons de poser les questions suivantes au sujet desquelles nous vous serions reconnaissants de nous faire connaître votre réponse $[. ..] 1^{\circ}$ - Le CAEE peutil continuer son action sur les bases préalablement établies? $2^{\circ}$ - Le Comité d'Accueil peut-il [...] ? $3^{\circ}$ - Le Comité d'Accueil peut-il [...] ? ${ }^{31}$ »

\section{Les raisons de la déférence : aide humanitaire et solidarité}

Une telle soumission étonne. Pour la comprendre, il faut considérer le contexte global dans lequel se trouve la CGT face à la guerre civile. Le soutien au camp républicain est évident, et consensuel. Mais, comme le reste de la gauche française (hormis le monde communiste), la CGT se divise au sujet de la non-intervention. Cette dernière la met en effet devant un dilemme apparemment insoluble : faut-il donner la priorité à la solidarité antifasciste ou au pacifisme ${ }^{32}$ ? Or, l'aide aux enfants désamorce en partie le problème en permettant de soutenir la cause républicaine à travers un mode d'action humanitaire, donc potentiellement «neutre ${ }^{33}$ » politiquement. Elle répond donc, au moins ponctuellement, à la crise de conscience provoquée par la non-intervention. Dès lors, tout se passe comme si, à défaut de participer directement à la lutte militaire, la CGT palliait la non-intervention en prenant en charge des enfants « dont pour la plupart les pères accomplissent héroïquement leur devoir civique ${ }^{34}$ », action qui « aidera puissamment [ces derniers] à mener plus allègrement leur lutte libératrice ${ }^{35} \%$. Le CAEE considère dès lors " comme de son devoir de [continuer et développer son action] en accord absolu avec le gouvernement espagnol ${ }^{36}$ ». Cette notion de «devoir » produit une quasi-inversion du rapport de force humanitaire classique : c'est la CGT (et la gauche française en général) qui est débitrice du gouvernement espagnol qu'elle aide, et non l'inverse.

Le renversement, au moins symbolique, de la relation de pouvoir entre bienfaiteur et victime secourue explique tant l'attitude de déférence de la CGT que la légitimité incontestée du gouvernement espagnol à dicter les règles de la protection offerte aux enfants. Cette légitimité se fonde aussi sur sa capacité et sa compétence, jamais mises en doute, à prendre soin de la population civile. L'activisme en matière de protection sociale et de scolarisation, la modernité pédagogique sont des motifs stéréotypiques de l'image de la République espagnole, parangon de la civilisation et de la modernité face à la barbarie franquiste ${ }^{37}$. Pour choisir un exemple parmi tant d'autres, dans un reportage sur les «Visages de l’Espagne loyale », le journaliste René Davenay écrit : 
«Les crèches, les dispensaires foisonnent ; la misère est dépistée. [...] L'assistance sociale est multiforme. [...] La mortalité infantile était une des plaies de l'Espagne. Elle tend aujourd'hui à disparaître. Même note quant à l'instruction publique. L'Espagne, en 1926, comportait 52 p. 100 d'illettrés. [...] Aujourd'hui, Valence, en pleine guerre, a fondé des milliers d'écoles ${ }^{38}$.

L'aide humanitaire ne se justifie donc pas par l'éventuelle incompétence, voire l'incurie de l'État et des familles espagnoles. Le sens et les limites de l'aide étrangère sont parfaitement définis, lors d'une conférence internationale sur l'Aide à l'enfance espagnole organisée à Paris en juillet 1938, par la déléguée du Comité d'aide norvégien :

«Notre tâche [sic] n'est donc point d'enseigner au gouvernement espagnol comment organiser le secours, il le sait déjà, il l'a déjà organisé beaucoup mieux que nous, parce qu'il connaît le peuple et ses demandes. Notre tâche [sic] est d'aider les autorités espagnoles à avoir tout ce qu'il faut pour rendre effectif le secours ${ }^{39}$. »

\section{Des neuf Indications aux douze Points : les limites de la déférence}

La docilité du CAEE devant les exigences espagnoles n'est cependant pas sans limites. En mai 1937, suite à un remaniement ministériel, le ministère de l'Instruction publique dirigé par le communiste Jesús Hernández prend la direction de ce qui est devenu, en quelques mois, un réseau transnational d'hébergement d'enfants espagnols couvrant l'Espagne, la France, la Suisse, la Belgique, l'Angleterre et l'URSS. Ici comme ailleurs, le parti communiste espagnol applique les principes de normalisation et de centralisation de l'action publique. Des dispositions légales mettent fin, au mois d'août 1937, à l'« état de dispersion dans lequel se trouvent actuellement, dans la plupart des pays, les services d'aide aux enfants espagnols évacués de notre territoire à l'étranger ». Le ministère créée une Délégation espagnole à l'enfance évacuée (DEEE) désignée comme «unique entité responsable de tout ce qui se réfère à l'installation, organisation, éducation et inspection des groupes d'enfants espagnols résidant à l'étranger ${ }^{40} »$.

17 Afin d'harmoniser l'action du CAEE avec ce nouveau dispositif institutionnel, le responsable de la DEEE fraîchement arrivé à Paris, Juan Comas, propose un nouvel accord en douze points. Mais ces nouvelles demandes conduisent au bord de la rupture. Les douze points sont refusés. Au long des mois de septembre et octobre 1937, les relations s'enveniment au point que le ministre Jesús Hernández lui-même doit dire son fait au CAEE :

«[L]es douze points [qui ont été soumis] supposent le maximum de concessions que le gouvernement de l'Espagne peut faire quant à l'intervention dans le contrôle, éducation et surveillance des petits Espagnols qui résident à l'étranger. S'il n'en était pas ainsi, il serait porté atteinte au décret du 6 août dernier et à l'ordre ministériel du 10 du même mois et la DEEE n'aurait aucune raison d'être ${ }^{41}$.»

Que s'est-il passé? C'est en fait moins le contenu pratique et pédagogique des propositions espagnoles qui pose problème que la délimitation des responsabilités et, au final, l'équilibre du rapport de force entre les deux parties. Même si, en décembre 1936 et en avril 1937, le gouvernement espagnol avait fixé de manière plus ou moins unilatérale les conditions de l'aide humanitaire, le CAEE conservait toute autonomie pour la mener à bien sur le sol français. Les termes du contrat avaient été modifiés, mais pas son esprit. Désormais, le gouvernement espagnol veut diriger lui-même le dispositif créé par le CAEE, et faire de ce dernier son subordonné. Le délégué Juan Comas envoie ainsi le décret et l'ordre de création de la DEEE à toutes les colonies d'enfants espagnols en France, sans passer par le CAEE. Il se rend sans l'en aviser dans le camp provisoire du Val d'Or, près de 
Saint-Cloud, pour y prendre 26 enfants dont il a arrangé le placement à Marseille. Mais l'entrée au Val d'Or lui est refusée et, lors de sa réunion suivante, le 15 septembre 1937, le CAEE « reje[tte] [s]a pétition »- ce que le délégué espagnol « considère inadmissible ${ }^{42}$ ». En fait, la création de la DEEE retire à la CGT la délégation de pouvoir qui fondait son accord initial avec le gouvernement espagnol. Ceci est inacceptable pour une organisation plus puissante que jamais et à la longue tradition d'indépendance vis-à-vis des pouvoirs politiques et étatiques. Pour Georges Buisson, secrétaire adjoint de la CGT et secrétaire du CAEE, les lettres de Juan Comas sont "rédigées en des termes qu'il accepterait difficilement du gouvernement français lui-même ${ }^{43}$ ».

L'impasse est telle que, malgré le soutien de son ministre, le délégué espagnol se voit obliger, en octobre 1937, de mettre de l'eau dans son vin et d'édulcorer les douze points initialement imposés. À chaque paragraphe, la nécessité de la "collaboration» vient remplacer la «reconnaissance du droit de la Délégation à intervenir ${ }^{44}$ ». Que l'esprit d'indépendance de la CGT ou la volonté de contrôle espagnole soit en cause, quelques semaines plus tard, le gouvernement républicain décide d'interrompre les évacuations d'enfants à l'étranger ${ }^{45}$. Elles ne reprennent vers la France qu'après un nouveau changement ministériel, qui voit l'Instruction publique passer aux mains d'un ministre anarchiste, en avril 1938.

\section{La mise en œuvre du contrat humanitaire}

\section{"Ménager les transitions ${ }^{46}$ »}

La nature contractuelle et négociée de l'action humanitaire de la CGT se reflète dans le dispositif mis en œuvre à l'attention des enfants. Loin de profiter de l'immixtion humanitaire pour modifier ou "civiliser » les mœurs de ceux dont elle a la charge, elle s'efforce de maintenir leurs liens avec leur culture, leur passé, leur famille, en somme tout ce qui est compris comme participant de leur identité. En cela, elle est, à nouveau, en complet accord avec le gouvernement espagnol. À partir du moment où le séjour des enfants doit être temporaire (jusqu'à la victoire républicaine que l'on espère prochaine), « il ne peut être question d[e les] adopter [...]. Rien ne doit être fait pour détourner ces enfants de leur nationalitée ${ }^{47} »$. Ceux-ci ne sont donc jamais considérés comme des enfants abstraits et sans attaches. Bien au contraire, le CAEE prend en compte leur différence culturelle, quitte à verser dans le stéréotype : «Sans doute, ils ont la belle fierté de leur race ; les méthodes coercitives n'arrivent qu'à les heurter. Ils se cabrent devant la menace et se rebellent contre les punitions ${ }^{48}$ "; « Comme tous ceux de leur race, ces enfants sont musiciens, artistes ${ }^{49}$.» Leur langue, leur caractère, leurs opinions politiques sont valorisés : «Bientôt, ils arrivent en gare du Nord et voici qu'ils descendent et se remettent en rangs en chantant l'Internationale dans la langue espagnole, leur langue maternelle. Et lorsque le photographe les prend, tous les petits poings se dressent ${ }^{50}$.» Dès lors, «il ne faut pas qu'ils se trouvent brusquement transplantés dans un climat différent de celui auquel ils ont coutume; il leur faut, d'autre part, une adaptation progressive à la langue française, qui ne leur est pas familière ${ }^{51} »$.

21 La question du climat est en effet prise très au sérieux. Les évacuations ayant commencé au début de l'hiver 1936-1937, les lieux d'hébergement sont jusqu'à la fin du printemps exclusivement localisés « dans [la] région méridionale, attendant des temps plus sereins pour les autres départements ${ }^{52} »$. Les premiers camps provisoires sont situés dans les 
Pyrénées-Orientales, l'Isère, la Haute-Loire, l'Hérault, les Basses-Pyrénées. Les premiers placements définitifs en famille se font dans les Pyrénées-Orientales, le Dauphiné, l'Aude. On n'envisage d'envoyer en Hollande, en Belgique et en Suisse que «les enfants les plus robustes $^{53}$ ». Si l'on suit les nouvelles publiées dans Le Peuple, ce n'est qu'à l'arrivée du printemps 1937 qu'un premier groupe d'enfants est placé en région parisienne ${ }^{54}$. La nécessité de ménager des transitions conduit également le CAEE à créer des « camps de triage " dans lesquels les enfants doivent rester un mois pour subir des examens médicaux et s'adapter à leur nouvel environnement. Ces camps de triage révèlent la prise en compte, par le CAEE, des souffrances physiques et morales subies par les enfants du fait de la guerre et du déplacement : «Ce stage de transition se montre indispensable. Les souffrances des petits ont été grandes et leur état s'en ressent ${ }^{55}$. » Les camps de triage donnent aussi le temps d'organiser les placements, en essayant de régler l'épineux problème des fratries "qu'il serait cruel de séparer ${ }^{56}$ ». Une fois encore, le CAEE ne se contente pas de mots, et les recensements des enfants hébergés en France donnent toute la mesure de l'effort fourni en ce sens. Sur un échantillon de 615 enfants appartenant à 257 fratries, quinze enfants tout au plus (appartenant à onze fratries différentes) se trouvent dans un département différent de leurs frères et sœurs. Parmi les 246 fratries hébergées intégralement dans le même département, et qui regroupent 585 enfants, 28 au plus ne sont pas hébergés dans la même commune que leurs frères et sœurs. En revanche, les quatre enfants Aval sont logés à Salaise-sur-Sanne en Isère, les trois Bascaran Oleaga à Allevard-les-Bains ${ }^{57}$, etc.

Enfin, le CAEE ne se cache pas les complications nées du problème de la langue. Ainsi, dans les neuf indications convenues initialement avec le ministère de la Santé et de l'Assistance sociale il est prévu que «les organisations du corps enseignant [français] mettront à la disposition du comité d'accueil des professeurs parlant l'espagnol, de façon à obtenir une plus rapide adaptation des enfants ${ }^{58} »$. Mais de telles compétences sont parfois difficiles à trouver. Ainsi lors de l'arrivée, en juillet 1937, d'un groupe de 40 enfants à Montbéliard, "les membres du comité [...] aidés par deux institutrices espagnoles qui, hélas! ne parlaient pas un mot de français, s'attachèrent à placer dans la même localité les enfants de même famille ${ }^{59}$ ». Malgré ces difficultés, comme on le voit, la présence d'institutrices espagnoles venues avec les enfants, l'attention à l'intégrité des fratries, tout est fait pour faciliter l'adaptation des enfants à un monde étranger. Car ce séjour en France ne doit pas être une simple parenthèse. L'institutrice française Marcelle Charaud, directrice suppléante de la colonie de Chantilly, le clame :

«Peut-on accepter que le séjour en France de ces enfants même écourté, ne laisse en eux aucune trace?»

«Leur apprendre notre langue, avec la leur, avant l'âge des métiers? N'est-ce pas notre devoir ${ }^{60}$ ?»

$\mathrm{Au}$ final, le dispositif d'hébergement du CAEE reflète bien l'esprit de collaboration qui imprègne tout le projet. Les responsables du CAEE essaient en effet de semer les graines d'une future amitié franco-espagnole: «Dans notre Pays ils apprennent à connaître la véritable figure de la France démocratique et hospitalière. Pour l'avenir cet amour de la seconde patrie ne peut que resserrer les liens séculaires qui unissent deux pays amis ${ }^{61}$. " Pour les familles, les syndicalistes et les pédagogues français, les enfants espagnols ne doivent pas vivre isolés dans des îlots d'hispanité. Sans préjudice de leur culture antérieure, ils doivent connaître la langue, la culture, les paysages de la France. Ce n'est pas, cependant, la priorité du gouvernement espagnol, surtout à partir du remaniement ministériel de mai 1937 et la création de la DEEE au mois d'août de la même année. 


\section{L'éducation nationale en exil} de l'Assistance sociale en décembre 1936 ne porte sur l'éducation des enfants espagnols en France. Puisqu'ils doivent être placés en famille, ils suivront l'école primaire française. Le décret qui signe la reprise en main par le ministère de l'Instruction publique des enfants évacués à l'étranger fait de l'éducation, en revanche, un souci majeur. La nouvelle priorité est de donner aux enfants espagnols une éducation espagnole, en espagnol, par des Espagnols, pour s'assurer qu'ils restent bien des Espagnols. Ces enfants ne doivent donc plus être confiés à des familles françaises, mais vivre en collectivité dans des colonies d'enfants car :

« le ferme critère [est] que les enfants évacués à l'étranger à cause des nécessités de la lutte reçoivent tous, dans la mesure du possible, l'éducation et l'enseignement sous la direction de maîtres espagnols afin que [...] ils ne perdent pas ce qui les unit avec la culture espagnole ni surtout, la connaissance de leur langue maternelle... ${ }^{62}$ »

Ce tournant pédagogique et nationaliste est indissociablement lié à la volonté de reprise en main et de contrôle du gouvernement espagnol analysée plus haut. Comme le précise le nouveau délégué au CAEE, «le gouvernement d'Espagne désireux de maintenir des rapports ininterrompus avec tous les enfants espagnols évacués à l'étranger, considère pour accomplir son devoir de tutelle et de contrôle - que ses colonies doivent être dirigées par du personnel enseignant espagnol ${ }^{63}$ ».

Si cette nouvelle politique provoque des tensions entre le CAEE et le gouvernement espagnol du point de vue de la répartition des pouvoirs, de nombreux pédagogues français partagent cette conception nationaliste de l'enfant ainsi que l'intérêt pour le potentiel pédagogique de la vie en collectivité ${ }^{64}$. Alors que le placement familial est plus économique et facile à mettre en œuvre et qu'elle manque dramatiquement de fonds, la section des Basses-Pyrénées du Syndicat des instituteurs se refuse en janvier 1938 à distribuer dans des familles les 480 enfants de sa colonie de Bayonne. Elle s'en explique avec éloquence à ses adhérents :

«Que faire? Répartir ces enfants dans les familles? Non. Aimez-vous l'Espagne et voulez-vous lui rendre de bons petits Espagnols et non des petits étrangers? Laissez ces enfants dans la Colonie. [...] Et demain, l'âme toute pénétrée du génie espagnol le plus noble tout auréolé par le grand sacrifice des héros, ces enfants travailleront avec joie et enthousiasme au relèvement de l'Espagne meurtrie. [...] Ensemble, les petits réfugiés vivent en Espagnols et ce sont des Espagnols bien pénétrés du génie de la race qu'il faudra rendre à leur Patrie pour qu'ils en assurent le relèvement et en dirigent les destinées ${ }^{65}$.»

\section{Conclusion}

L'évacuation des enfants espagnols navigue, selon les moments et les acteurs, entre la logique humanitaire de l'aide gratuite de la France à l'Espagne, celle de la solidarité entre semblables, et les tentatives de projection sur le sol français du proto État providence espagnol, qui ferait de la CGT une sorte de service public délégué de cet État - une option inacceptable pour elle. Le rapport de force entre bienfaiteur et secouru est donc un enjeu central, mais il est moins une donnée structurelle liée au déséquilibre provoqué par un don qu'un enjeu sans cesse renégocié. L'affirmation par l'État espagnol de sa souveraineté

Revue d'histoire de l'enfance « irrégulière », 15 | 2013 
sur ses nationaux y est pour beaucoup. Mais tout aussi important est que cette dernière soit reconnue par les dirigeants de la gauche française qui conçoivent leur action comme la réciproque du sacrifice d'une République espagnole fantasmée, dont la lutte serait menée au nom de et au bénéfice de tous contre le fascisme. Joue enfin l'ethos particulier de la solidarité identitaire (de classe), aspect fondamental de la pratique syndicale, fondant une relation d'entraide entre égaux.

L'historiographie de l'humanitaire définit généralement son objet en termes d'acteurs, et non d'actions ${ }^{66}$. Ceci conduit à une relative cécité devant les opérations humanitaires menées par des organisations qui ne poursuivent pas, en temps normal, ces buts - comme la CGT. La plupart des travaux portent sur les acteurs institutionnels (Société des Nations, Nations unies) et sur les grandes organisations estampillées «humanitaires». Or, l'humanitaire relève peut-être davantage d'un mode d'action que d'un type d'acteur ou d'une "posture idéologique ${ }^{67}$ ». Il est permis de supposer que si l'on change de point d'entrée, son histoire pourrait s'en trouver à la fois enrichie et complexifiée.

\section{NOTES}

1. Le gouvernement espagnol comptabilise, en mars 1938, 7305 enfants hébergés en France sous son contrôle. De nombreuses organisations concourent à leur soutien, de l'union des syndicats de la Marne au Comité suédois d'aide à l'Espagne. Mais toutes sont supervisées, de près ou de loin, par le CAEE, c'est-à-dire la CGT. Fontainebleau, Centre des Archives Contemporaines (ci-après CAC), Fonds de la Commission d'aide aux enfants espagnols réfugiés en France, 20010221/9, inventaire 2, dossier 2, f. 66-76, Rapport que la Délégation espagnole pour l'enfance évacuée présente à la conférence organisée par le Comité international de coordination et d'aide à l'Espagne républicaine, Paris, 20-21 novembre 1937. Annexe III et Supplément $n^{\circ} 1$.

2. ZAHRA Tara, Kidnapped Souls: National Indifference and the Battle for Children in the Bohemian Lands, 1900-1948, Ithaca/London, Cornell University Press, 2008, XII-279 p.

3. Nous reprenons bien sûr cette expression de THIESSE Anne-Marie, Faire les Français: quelle identité nationale?, Paris, Stock, 2010, 194 p.

4. En dehors de l'immense bibliographie anglo-saxonne sur l'opération Pied Piper, voir Downs Laura Lee, "Les évacuations d'enfants en France et en Grande-Bretagne (1939-1940) », Annales. Histoire, Sciences Sociales, 2, 2011, p. 413-448, qui fait également référence aux évacuations enfantines de 1918. Sur des épisodes moins connus de l'entredeux-guerres : FISCHER Conan, The Ruhr crisis, 1923-1924, Oxford, Oxford University press, 2003, p. 117-135 ; SHARPLES Caroline, "Kindertransport : Terror, Trauma and Triumph", History Today, vol. 60, 3, 2004, p. 23-29. Nous nous permettons également de renvoyer au premier chapitre de notre thèse en cours intitulée «L'évacuation et l'accueil des enfants espagnols en France: cartographie d'une mobilisation transnationale (1936-1942)», menée à l'EHESS sous la direction de Laura Lee Downs. 
5. BRODIEZ Axelle, DUMONS Bruno, "Éditorial: Faire l'histoire de l'humanitaire", Le mouvement social, 227, 2009/2, p. 3-8.

6. RYFMAN Philippe, Une histoire de l'humanitaire, Paris, la Découverte, coll. «Repères ", 2008, p. 11. Une exception salutaire à cette myopie dans BECKER Annette, Oubliés de la Grande Guerre : humanitaire et culture de guerre, 1914-1918 : populations occupées, déportés civils, prisonniers de guerre, Paris, Hachette Littérature, 2003, 395 p.

7. WATENPAUGH Keith David, "The League of Nations' Rescue of Armenian Genocide Survivors and the Making of Modern Humanitarianism, 1920-1927», The American historical review, vol. 115, 5, 2010, p. 1315-1339. Voir également les numéros spéciaux récemment consacrés à ce thème dans deux revues françaises, et qui traitent tous deux de l'avant 1945 : BRodiEZ Axelle, DUmons Bruno (dir.), Le Mouvement Social, « Faire l'histoire de l'humanitaire ", 227, 2009/2, 122 p. ; et BOssUAT Gérard, Matériaux pour l'histoire de notre temps, «Les humanitaires européens au $\mathrm{XX}^{\mathrm{e}}$ siècle : entre urgence et diplomatie », 3, 2009, $94 \mathrm{p}$.

8. BECKER, Oubliés de la Grande Guerre..., p. 155-157.

9. MARShall Dominique, "The Construction of Children as an Object of International Relations: The Declaration of Children's Rights and the Child Welfare Committee of League of Nations, 1900-1924 », International Journal of Children's Rights, 7, 1999, p. 134.

10. WATENPAUGH, «The League of Nations' rescue... » Ceci va tout à fait dans le sens des observations du sociologue Didier Fassin, qui montre combien l'inégalité du rapport de force au sein de la relation humanitaire est structurelle et, en ce sens, lui est inhérente : "Le problème n'est toutefois pas psychologique, ni même éthique [...] : il est strictement sociologique. Ce n'est pas la condescendance éventuelle de l'aidant qui est en cause, pas plus que la signification de son acte d'aider, ce sont les conditions du rapport social liant les deux parties qui, au-delà de toute intention des agents, font de la compassion un sentiment moral sans réciprocité possible.» FASSIN Didier, La raison humanitaire: une histoire morale du temps présent, Paris, Gallimard, coll. « Hautes études », 2010, p. 11.

11. "Idiots and cretins abound", ZAHRA Tara, The Lost Children: Reconstructing Europe's Families After World War II, Cambridge/Londres, Harvard University Press, 2011, p. 40.

12. Selon les mots de la quaker britannique Francesca Wilson au sujet des familles françaises auprès desquelles elle travaille. Ibid., p. 28.

13. Précisons que, dans le cas qui nous occupe, les acteurs qualifient eux-mêmes systématiquement leur action d'«humanitaire ». Pour une brève discussion de leurs usages de cette catégorie, nous nous permettons de renvoyer à KEREN Célia, «Niños víctimas con el puño en alto. Los niños españoles evacuados a Francia durante la Guerra de España ", TRUC Gérome (dir.), Víctimas políticas en España y Europa, Madrid, Éditions de la Casa de Velázquez, à paraître en 2013.

14. Une première tentative d'évacuation organisée d'enfants est portée, dès le mois d'août 1936, par l'Association nationale du soutien de l'enfance, héritière du Secours ouvrier international. Elle est ensuite relayée par le Secours rouge/populaire de France, qui soutient un groupe d'enfants évacués d'Irun et hébergés dans une institution près de Biarritz. La fondation du CAEE et la reconnaissance par le mouvement international d'aide à l'Espagne de son monopole sur les évacuations d'enfants vers la France font donc l'objet d'une lutte assez âpre, remportée par ce dernier. Le CAEE constitue dès lors l'accueil des enfants espagnols en domaine quasi réservé de la gauche non communiste et 
donc, au sein de la CGT, des ex-confédérés. À ce sujet, voir le chapitre 2 de notre thèse en cours, «L'évacuation et l'accueil... »

15. Sur la création du CAEE et son identification avec la CGT, voir KEREN Célia, «Les défis de la solidarité internationale : La collaboration entre la Ligue des Droits de l'homme et la Confédération générale du travail au sein du Comité d'accueil aux enfants d'Espagne (1936-1939) ", DARD Olivier, SÉVILLA Nathalie (dirs.), Le phénomène ligueur en Europe et Amérique du Nord, Metz, Presses Universitaires de Metz, 2011, p. 269-286.

16. Ibid. Cet accord est assorti d'une certaine bienveillance, y compris des autorités locales, au moins jusqu'à l'éclatement du Front populaire en 1938. Le gouvernement français laisse cependant aux seules organisations privées la gestion du programme d'évacuation. Il n'est sollicité que sur des points réglementaires précis.

17. "Indications pour la venue en France des enfants d'Espagne", La Voix du Peuple. Bulletin officiel de la Confédération générale du travail (ci-après LVP), décembre 1936, p. 793.

18. Montreuil, Institut d'Histoire sociale de la CGT (ci-après IHS-CGT), Fonds de la CGT rapatrié de Russie, Activités internationales, 97CFD45/6, «Indications à suivre pour la venue des enfants Espagnols en France ", document manuscrit, sd, f. 15.

19. "Circulaire H. 76 de Georges Buisson destinée aux Fédérations nationales et aux Unions départementales: "Pour Aider l'Espagne!" Paris, 29 décembre 1936 ", LVP, décembre 1936, p. 791-792.

20. «Indications pour la venue en France des enfants d'Espagne », LVP, décembre 1936, p. 793.

21. «L'effort de solidarité doit continuer pour les petits orphelins espagnols. Sous l'égide de la CGT le Comité d'Accueil adresse à tous un pressant appel », Le Peuple. Organe officiel de la Confédération Générale du Travail (ci-après Le Peuple), 5882, 27 février 1937, p. 4.

22. "La CGT à la radio. L'accueil aux enfants d'Espagne », Le Peuple, 5816, 23 décembre 1936, p. 2.

23. «L'accueil aux Enfants d'Espagne », LVP, juillet 1937, p. 457-466.

24. «L'accueil aux Enfants d'Espagne », LVP, août 1938, p. 555-557.

25. Montreuil, IHS-CGT, Fonds de la CGT rapatrié de Russie, Activités internationales, 97CFD45/6, Copie de la lettre de Georges Buisson, secrétaire-adjoint de la CGT, à Julien Forgues, secrétaire de l'Union départementale des syndicats de Haute-Garonne, Paris, 5 janvier 1937, f. 9 ; et copie de la lettre de Georges Buisson au Comité d'aide au peuple espagnol, Paris, 24 décembre 1936, f. 21.

26. Fontainebleau, CAC, Fonds de la Commission d'aide aux enfants espagnols réfugiés en France, 20010221/9, inventaire 2, dossier 3, f. 179, Lettre de Federica Montseny au CAEE, Valence, 8 avril 1937 : «Los acuerdos anteriores respondían entonces al criterio del Gobierno : actualmente éste lo ha modificado en el sentido de aceptar solo el régimen de Colonias para los niños evacuados en el extranjero ".

27. Ibid., f. 181-186, copie de la lettre de Georges Buisson, secrétaire du CAEE, au ministère de la Santé et de l'Assistance sociale à Valence, sd [Paris, 31 mars 1937].

28. Ibid., f. 179, lettre de Federica Montseny au CAEE, Valence, 8 avril 1937: «En este momento preferimos disminuir el número de envios que no [sic] colocarlos en régimen familiar ».

29. FARRÉ Sebastián, « Política y propaganda : niños refugiados en Suiza durante la Guerra Civil », Alted Alicia, GONZÁLEz Roger et José millán Maria (dir.), El exilio de los niños, Madrid, Fondation Francisco Largo Caballero, Fondation Pablo Iglesias, 2003, p. 117. 
30. Montreuil, IHS-CGT, Fonds de la CGT rapatrié de Russie, Activités internationales, 97CFD45/6, f. 15-16, Lettre de Suzanne Blum, pour le Arbeitsgemeinschaft für Spanienkinder, à Georges Buisson, secrétaire général du CAEE, Berne, 12 juin 1937.

31. Fontainebleau, CAC, Fonds de la Commission d'aide aux enfants espagnols réfugiés en France, 20010221/9, inventaire 2, dossier 3, f. 181-186, Copie de la lettre de Georges Buisson, secrétaire du CAEE, au ministère de la Santé et de l'Assistance sociale à Valence, s. d. [Paris, 31 mars 1937].

32. LABORIE Pierre, «Espagnes imaginaires et dérives pré-vichystes de l'opinion française, 1936-1939", SAGNeS Jean, CAUCANAS Sylvie (dir.), Les Français et la guerre d'Espagne, Perpignan, Presses Universitaires de Perpignan, 1990, p. 93.

33. L'idée de neutralité est fondamentale au développement de l'humanitaire, notamment en direction des enfants. MARSHALL, "The Construction of Children... », p. 138-139, art. cit. Sur le rapport entre aide aux enfants et non-intervention, voir KEREN Célia, « Les enfants espagnols réfugiés en France : 1939 ou la crise de la solidarité ouvrière », Cahiers du Centre de recherches historiques, 44, octobre 2009, p. 75-89.

34. "Circulaire H. 76 de Georges Buisson destinée aux Fédérations nationales et aux unions départementales: "Pour Aider l'Espagne!" Paris, 29 décembre 1936 ", LVP, décembre 1936, p. 791-792.

35. Ibid.

36. Fontainebleau, CAC, Fonds de la Commission d'aide aux enfants espagnols réfugiés en France, 20010221/9, inventaire 2, dossier 3, f. 181-186, Copie de la lettre de Georges Buisson, secrétaire du CAEE, au ministère de la Santé et de l'Assistance sociale à Valence, s. d. [Paris, 31 mars 1937].

37. En cela, la propagande républicaine perpétue de manière frappante la culture de guerre de 14-18. Voir AUDOIN-ROUZEAU Stéphane et BECKER Annette, 14-18, retrouver la guerre, Paris, Gallimard, 2000, $272 \mathrm{p}$.

38. Davenay René, «IV. Valence, au cœur de l'Espagne loyale», Le Peuple, 6131, 3 novembre 1937, p. 1.

39. Fontainebleau, CAC, Fonds de la Commission d'aide aux enfants espagnols réfugiés en France, 20010221/8, dossier 305, f. 27-29, Rapport de Nini Haslund, déléguée du Comité d'aide de Norvège et de l'office international pour l'enfance à Valence, «Le travail de secours international. Les dispensaires ».

40. Ibid., 20010221/9, inventaire 2, dossier 2, f. 88-89, brochure du ministère de l'Instruction publique: "Délégation Espagnole pour les Enfants évacués. Paris", contenant le décret de la présidence du Conseil des ministres du 6 août 1937 et l'ordre du ministère de l'Instruction publique du 10 août 1937.

41. Ibid., f. 145-146, communication officielle de Jesús Hernández, ministre de l'Instruction publique, à Juan Comas, délégué espagnol à l'enfance évacuée, Valence, 15 octobre 1937, transmise au CAEE le 20 octobre 1937.

42. Ibid., f. 116-119, lettre de Juan Comas, délégué espagnol à l'enfance évacuée, à Georges Buisson, secrétaire du CAEE, Paris, 17 septembre 1937.

43. Ibid., f. 131, Copie de la lettre de Georges Buisson, pour le CAEE, à Juan Comas, le 30 septembre 1937.

44. Ibid., f. 148-149, Note de la DEEE à l'intention du CAEE, Paris, 25 octobre 1937. 
45. Nanterre, Bibliothèque de documentation internationale et contemporaine, Archives de la Ligue des Droits de l'homme, F $\Delta$ rés. 798/61, dossier 3, f. 95-107, Bulletin d'Information du Comité international de coordination et d'information pour l'aide à l'Espagne républicaine (CICIAER), 15 janvier 1938.

46. « L'accueil aux enfants d'Espagne », LVP, juillet 1937, p. 457-466.

47. LAPIERRE Georges, « Pour les enfants de nos camarades instituteurs espagnols », L'École Libératrice. Organe hebdomadaire du Syndicat National des Institutrices et Instituteurs publics de France et ses Colonies, 8 e année, 17, 30 janvier 1937, p. 404.

48. «L'accueil aux Enfants d'Espagne », LVP, juillet 1937, p. 457-466.

49. ALM, "Un admirable exemple de solidarité. Le Comité d'Accueil aux Enfants d'Espagne ", Messidor. Hebdomadaire de la démocratie syndicale, 12, vendredi 3 juin 1938, p. 21.

50. JOUENNE Alice, «Chassés du nid. L'émouvant exode des enfants d'Espagne », Le Peuple, 5972, 28 mai 1937, p. 1.

51. "Circulaire H. 76 de Georges Buisson destinée aux Fédérations nationales et aux unions départementales: "Pour Aider l'Espagne!" Paris, 29 décembre 1936 ", LVP, décembre 1936, p. 791-792.

52. BUISSON Georges, «Dimanche 4 avril. Journée nationale de solidarité pour les enfants d'Espagne », Le Peuple, 5907, 24 mars 1937, p. 1.

53. «La CGT à la radio. L'accueil aux enfants d'Espagne. Causerie faite par Georges Buisson au poste de la Tour Eiffel », Le Peuple, 5886, 3 mars 1937, p. 2.

54. « Comment à Valence on considère le projet de trêve ou de médiation. "Pas de paix de Vergara!" », Le Peuple, 5970, 26 mai 1937, p. 1.

55. Cf. note 53.

56. «L'accueil aux enfants d'Espagne », LVP, juillet 1937, p. 457-466.

57. Alcalá de Henares, Archivo General de la Administración, Fondo Presidencia, (09)51/21124. "Relación de niños españoles con indicación del Departamento en que fueron alojados en Francia », sans date. Nous précisons «tout au plus » car nous ignorons le lieu (département et/ou commune) de résidence de certains enfants. Ainsi, sur les onze fratries que nous considérons comme ne résidant pas intégralement dans le même département, dans trois cas, nous considérons la fratrie comme séparée car nous ignorons où se trouve l'un de ses membres - il pourrait aussi bien être, en fait, dans le même département. Dans quatre autres cas sur les onze, un seul enfant est isolé d'une fratrie supérieure ou égale à trois individus (et dont les autres membres résident bel et bien dans le même département). Bien évidemment, la distance peut être grande au sein d'un département, et même s'ils sont placés dans une même commune, le sentiment de séparation des frères et sœurs peut être important. Nous nous intéressons ici uniquement aux objectifs du CAEE, et non au vécu des enfants.

58. «Indications pour la venue en France des enfants d'Espagne », LVP, décembre 1936, p. 793.

59. «Dans le Doubs. Une émouvante réception des enfants d'Espagne à Montbéliard », Le Peuple, 6022, 17 juillet 1937, p. 5.

60. BISO Jean, «Avec les enfants réfugiés espagnols. Au centre d'accueil de Chantilly », Le Peuple, 6059, 23 août 1937, p. 1. 
61. Fontainebleau, CAC, Fonds de la Commission d'aide aux enfants espagnols réfugiés en France, 20010221/9, inventaire 2, dossier 3, f. 13, Lettre d'Élie Sermet, secrétaire général de l'union départementale des syndicats de l'Aude, au préfet de l'Aude, 19 juillet 1938.

62. Ibid., dossier 2, f. 88-89, brochure du ministère de l'Instruction Publique : « Délégation Espagnole pour les Enfants évacués. Paris ", contenant le décret de la présidence du Conseil des ministres du 6 août 1937 et l'ordre du ministère de l'Instruction publique du 10 août 1937.

63. Ibid., f. 93-96, Copie de la lettre de Juan Comas, délégué espagnol à l'enfance évacuée, au CAEE, Paris, 30 août 1937.

64. Sur le débat entre les mérites respectifs du placement familial et du régime collectif, et le consensus final autour de la valeur pédagogique de ce dernier, voir Downs Laura Lee, Histoire des colonies de vacances de 1880 à nos jours, Paris, Perrin, 2009, 433 p.

65. J. L., « Un sou par jour », Bulletin du Syndicat national des instituteurs. Section des BassesPyrénées, 43, janvier 1938.

66. Par exemple RYFMAN Philippe, « Non-Governmental Organizations: an Indispensable Player of Humanitarian Aid », International Review-Red Cross-New Series, 2007, 89/865.

67. BRODIEZ Axelle, Le Secours populaire français, 1945-2000: du communisme à l'humanitaire, Paris, Presses de la Fondation nationale des sciences politiques, 2006, p. 37.

\section{RÉSUMÉS}

Pendant la guerre d'Espagne (juillet 1936-avril 1939), trente mille enfants espagnols sont évacués de la zone républicaine vers des pays étrangers, dont environ la moitié vers la France. Ils y sont pris en charge, hébergés dans des familles ou des colonies collectives, et scolarisés. Mais cet éloignement risque de distendre les liens entre l'État-nation espagnol et «ses » enfants. Il fait aussi surgir le spectre de leur "dénationalisation ». Dès lors, la relève de l'État providence espagnol par l'aide humanitaire ne se fait pas à n'importe quelles conditions. Cet article analyse la négociation par le gouvernement espagnol et la Confédération générale du travail de l'évacuation des enfants espagnols vers la France, et les implications pratiques de cet aspect négocié dans les choix des lieux, des structures d'accueil et des solutions pédagogiques. Ce cas amène à repenser la question du rapport de force entre bienfaiteur et victime secourue qui se joue dans l'action humanitaire, et des liens entre humanitarisme et solidarité dans les années 1930.

During the Spanish Civil War (July 1936-april 1939), 30.000 children were evacuated from the Republican zone to foreign countries, and about half of them went to France. There, they were taken care of, placed with French families or housed in children's homes, and educated. But the distance could jeopardize the relations between the Spanish Nation-State and "its" children. It also raised fears of denationalization. When humanitarian aid took over from the Spanish Welfare State, certain conditions had thus to be met. This article analyzes how the Spanish government and the French trade union negotiated the evacuation of Spanish children to France, and the practical implications of this negotiated dimension in the places, accomodation 
structures and pedagogical solutions that were chosen for the Spanish children. This example leads us to rethink the imbalance of power between the provider and receiver of aid, and the connections between humanitarianism and solidarity in the 1930s.

INDEX

Mots-clés : migrations forcées, enfants, Espagne, France, guerre civile, aide humanitaire

Keywords : forced migrations, children, Spain, France, civil war, humanitarian aid

\section{AUTEUR}

\section{CÉLIA KEREN}

Célia Keren est doctorante en histoire à l'École des hautes études en sciences sociales (EHESS), Paris, sous la direction de Laura Lee Downs. Sa thèse porte sur « L'évacuation et l'accueil des enfants espagnols en France : cartographie d'une mobilisation transnationale (1936-1942) ». Elle est membre-chercheur de l'École des hautes études hispaniques et ibériques (EHEHI) de la Casa de Velázquez, Madrid. 